\title{
URAGAN \& TRAGALDABAS: \\ two complementary approaches for the regular survey of Cosmic Rays
}

\author{
Juan A. Garzón for the TRAGALDABAS Collaboration* ${ }^{\dagger}$ \\ LabCAF, F. Física. Univ. of Santiago de Compostela \\ E-mail: juanantonio.garzon@usc.es \\ Igor I. Yashin \\ National Research Nuclear University MEPhI (Moscow Engineering Physics Institute), Moscow \\ E-mail: IIYashin@mephi.ru
}

\section{I.I. Astapov}

National Research Nuclear University MEPhI (Moscow Engineering Physics Institute), Moscow

\section{N.S. Barbashina}

National Research Nuclear University MEPhI (Moscow Engineering Physics Institute), Moscow

\section{A.N. Dmitriyeva}

National Research Nuclear University MEPhI (Moscow Engineering Physics Institute), Moscow

\section{A.A. Petrukhin}

National Research Nuclear University MEPhI (Moscow Engineering Physics Institute), Moscow

\section{V.V. Shutenko}

National Research Nuclear University MEPhI (Moscow Engineering Physics Institute), Moscow

Cosmic Rays are very useful for getting a real time information of many Earth's surrounding phenomena as solar activity, magnetic cloud detection and the high atmosphere dynamics, among others. In order to improve our knowledge in those fields, two singular and very different detectors installed in Europe, URAGAN in Moscow (Russia) and TRAGALDABAS in S. de Compostela (Spain), have decided to joint their efforts. Although they are based on different technologies and have very different performances, both of them offer very good angular resolution for muons of cosmic origin allowing a regular and coordinated survey of the arrival of low energy cosmic rays over a big region of the north hemisphere.

The 34th International Cosmic Ray Conference,

30 July- 6 August, 2015

The Hague, The Netherlands

\footnotetext{
*Speaker.

${ }^{\dagger}$ See full list of members of the TRAGALDABAS Colloboration at the end of the article
} 


\section{Introduction}

Cosmic Rays of a wide spectrum of energies are permanently arriving to Earth where they interact with the atmospheric atoms producing up to billions of secondary particles. Analyzing the rate and angular behavior in time of the most common particles, mainly neutrons and muons, at different latitudes, magnetic rigidity thresholds and energy cuts using absorbers, it is possible to make a regular survey of many phenomena with interest in the human activities and to issue precise forecasts for those phenomena: variations in the solar activity or the detection of intense geomagnetic storms, among others. For this purposes a wide networks of detectors, mainly neutron monitors and muons directional detectors, have been deployed over the Earth surface working in parallel and coordinating their efforts [1].

In this article we present two singular facilities, URAGAN, one of the component of the very big NEVOD research complex located at MEPhI ( (55.7 $\mathrm{N}, 37.7^{\circ} \mathrm{E}, 173 \mathrm{~m}$ a.s.1.), in Moscow (Russia), and TRAGALDABAS a new small size new generation tracking detector located at the Faculty of Physics of the USC (42.9 ${ }^{\circ}, 8.6^{\circ} \mathrm{W}, 240 \mathrm{~m}$ a.s.1.), in Santiago de Compostela (Spain). The distance between both facilities is $\sim 3557 \mathrm{~km}$, allowing them to look for common effects in the arrival of cosmic rays over different areas of the Earth's outer space asymptotic directions and anylize those effects in real time.
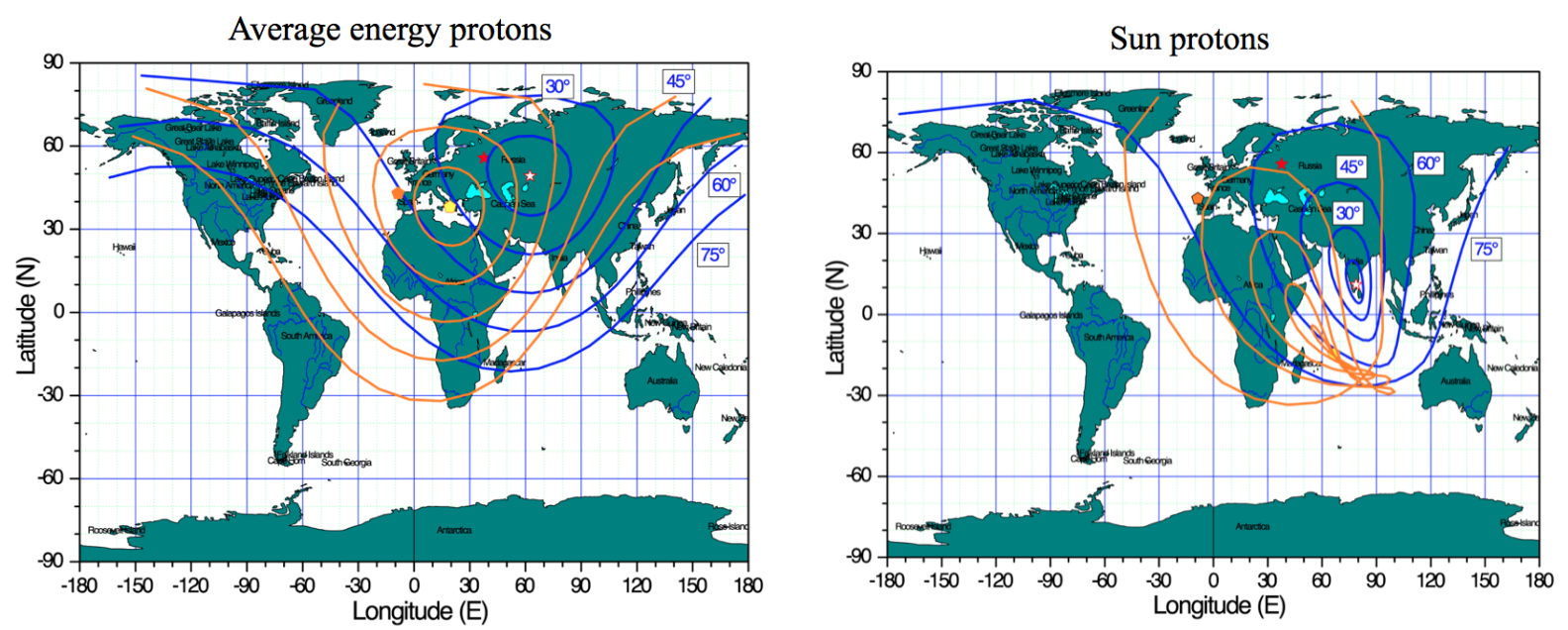

Figure 1: Aymptotic regions for different arrival angles of cosmic muons for both detectors. Blue contours correspond to URAGAN data and the yellow ones correspond to TRAGALDABAS.

As a consequence of their complementary performances and the distance existing between the detectors, URAGAN and TRAGALDABAS make up a very interesting tandem. As both detectors are relatively near in the same hemisphere, their joint operation may open plenty of new possibilities in the study of extraterrestrial effects related with cosmic rays. Fig. 1 show the asymptotic regions, covered by both detectors, for different arrival angles of muons produced by both average energy and solar protons. As shown in the figure, the very high overlap of the acceptances of both detectors would allow to improve significantly the angular resolution of the tandem as well as allowing to discriminate between common phenomena of interplanetary or solar origin and uncommon phenomena as the ones caused by local changes in the atmosphere conditions, in the magnetic 
field.

In section 2 we describe both detectors giving the most interesting features and performances of each of them. We also comment the advantages expected from the joint analysis of data of both detectors. In section 3 we present a joint preliminary analysis of the FD starting at June 22nd. 2015.

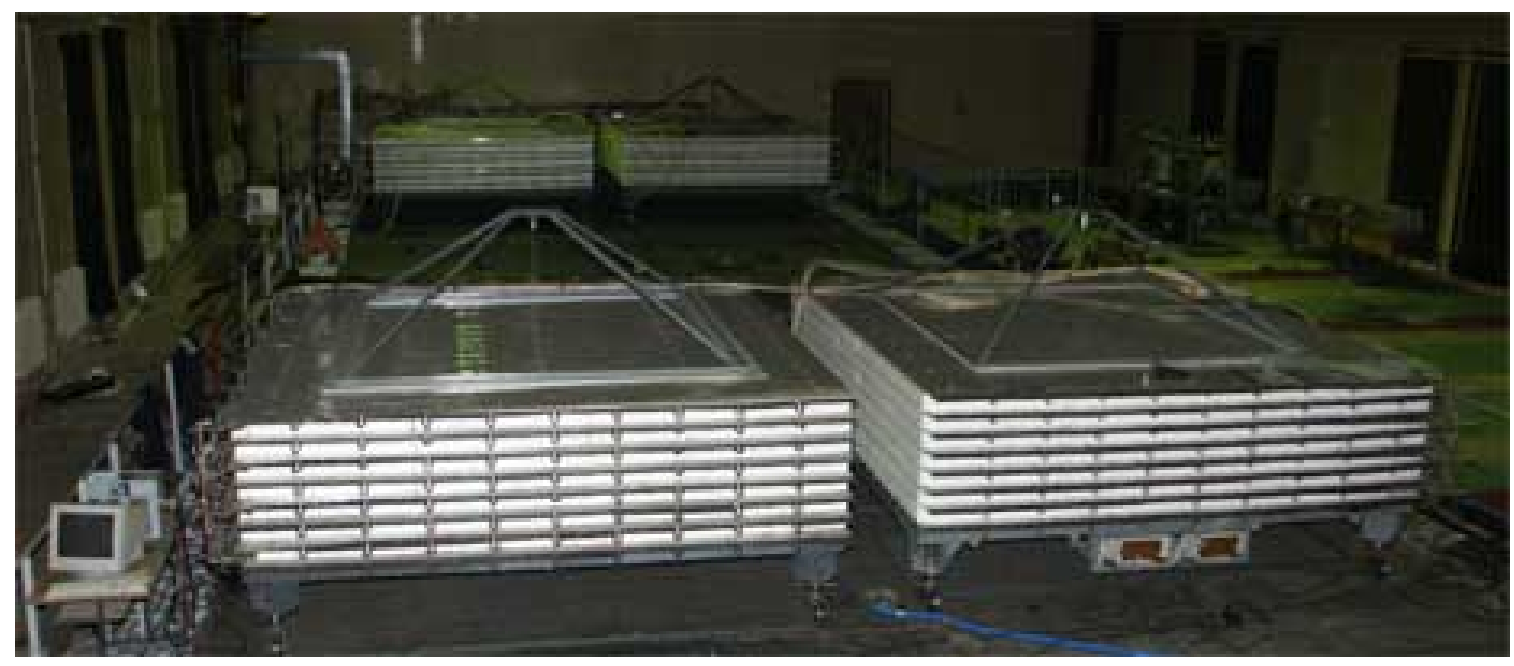

Figure 2: The muon hodoscope URAGAN on top of the NEVOD detector. The four Super Modules are easily distinguishable. Only three of them are taking data regularly. The forth one is used for testing and calibrating other detectors.

\section{The detectors}

\subsection{URAGAN}

Muon hodoscope URAGAN started taking regular data in 2006 and is a significative component of the NEVOD experimental complex [2]. It is composed by four super-modules, three of them are taking data permanently while the forth one is used for complementary calibration purposes (see Fig. 2). A supermodule (SM) is composed by eight planes of 320 streamer tubes with dimensionss $10 \mathrm{~mm} \times 10 \mathrm{~mm} \times 3500 \mathrm{~mm}$ each. The total area of one $\mathrm{SM}$ is $11.5 \mathrm{~m}^{2}$. The readout is performed with a $1.2 \mathrm{~cm}$ pitch by $320 \mathrm{Al}$ electrodes in the $\mathrm{X}$ oriented planes and $288 \mathrm{Al}$ electrodes in the $\mathrm{Y}$ oriented perpendicular direction. The height of each module is $50 \mathrm{~cm}$, being the maximum accesible zenith angle of $75^{\circ}$. A muon track is recorded if there is a coincidence between at least four $\mathrm{X}$ planes inside a time gate of $250 \mathrm{~ns}$. The energy threshold of the detected muons range between $200 \mathrm{MeV}$ and $600 \mathrm{MeV}$ for the different zenith angles. The scheme of a muon detection in a single SM is shown in Fig. 3. The track angular resolution is always better than $1^{\circ}$.

The mean counting rate of reconstructed muon tracks of each of the SM is $\sim 1300 \mathrm{~s}^{-1}$. The mean live time provided by the acquisition system is of $\sim 54 \mathrm{~s}$ per minute. Every minute three 90x90 cell matrices are stored with the number of arriving muons detected during last minute as a function of the following pair of variables: zenith and azimuthal angles, $\mathrm{X}$ and $\mathrm{Y}$ projections of zenith angle and, finally, the slopes of the $\mathrm{X}$ and $\mathrm{Y}$ projections of the zenith angle. These 1-min 
matrices are the basic information for most of the analysis related with the processes of cosmic ray modulation in the heliosphere and Earth's magnetosphere and atmosphere. Fig. 4 shows the matrix of the angular distribution of 1-minute of data taken with URAGAN.

Starting from the basic 1-min matrices, 5-minute, 60-minute and 24-hour matrices are also built for further purposes like solar activity analysis, atmospheric phenomena and others. Before using them, all the matrices are corrected to eliminate both pressure and temperature atmospheric effects. After the averaging, normalization and filtering procedures, every matrix represents the snapshot of upper hemisphere in "muon light" with a certain exposure (1, 5 or $60 \mathrm{~min})$. The analysis of the matrices' anisotropy provides information about the direction of cosmic ray modulation processes.

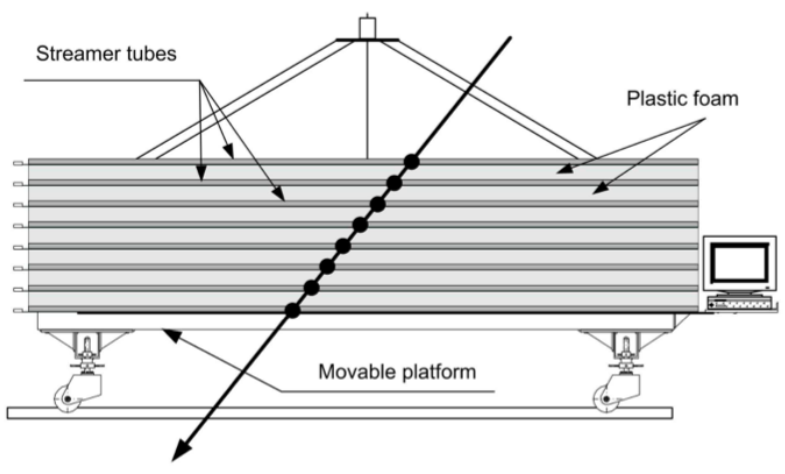

Figure 3: Scheme of a single particle detection in one Super Module of URAGAN

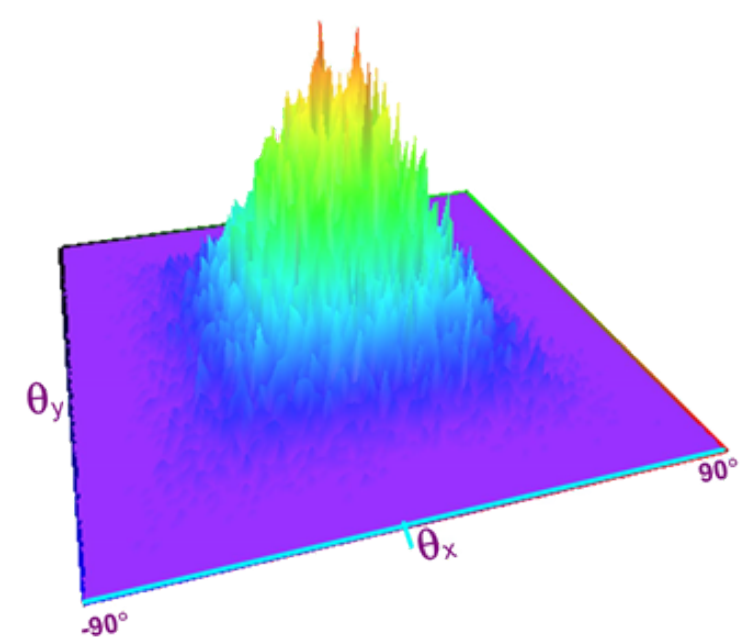

Figure 4: Example of one minute of data taken with three Super Modules of URAGAN

\subsection{TRAGALDABAS}

TRAGALDABAS is a new generation cosmic ray detector based on the RPC (Resistive Plate Chamber) technology based on the TRASGO concept [4]. It was developed to better understand several effects observed in the analysis of cosmic ray data taken during the commissioning of the RPC ToF wall of the HADES experiment at the GSI [5]. The detector started to take test data in Sept. 2013 with only 2 planes but it has undergone several improvements since then. In its present layout (see Fig. 5), the detector has been working rather smoothly since the end of March 2015. It covers a surface of $1.2 \times 1.5 \mathrm{~m}^{2}$ and has a height of $1.8 \mathrm{~m}$. It is composed of three planes of RPC cells readout by $120 \sim 11 \times 11 \mathrm{~cm}^{2}$ pads each, providing a time resolution of $\sim 300 \mathrm{ps}$. All this features does allow it to reconstruct the time-space microstructure of the front of the cosmic ray air showers with an angular resolution better than $2^{\circ}$ and an arrival time resolution better than 180ps for single tracks. Another uncommon performance is that the detector is sensitive to both the muonic and the electromagnetic (EM) components of the cosmic rays allowing to estimate the arrival of cosmic air showers having only EM component. Fig. 6 shows an example of the tracking capability of the detector. 


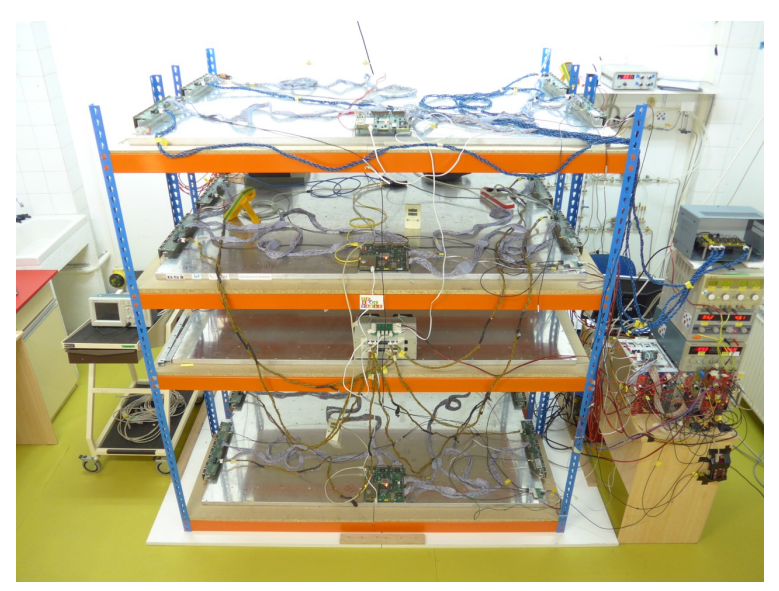

Figure 5: Present layout of the TRAGALDABAS detector. Starting from the top, only the 1st., 2nd. and 4th. planes are fully instrumented with the read-out electronics. Trigger is done by coincidences in planes 2nd. and 4th.

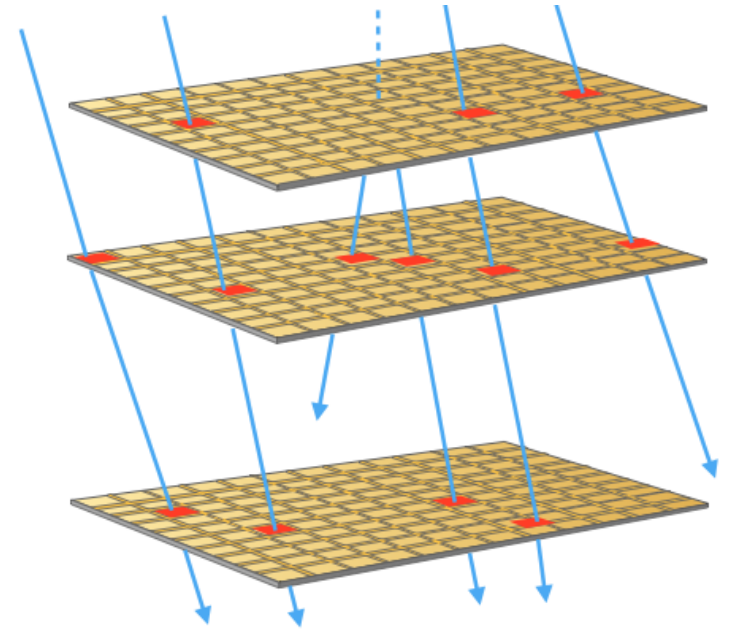

Figure 6: Example of the tracking performances of the TRAGALDABAS detector for a high multiplicity event. Electromagnetic component will be partially separated by software.

\section{Example of the joint performances of both detectors}

As an example of the joint capabilities provided by both detectors, we present the preliminary results of data obtained during a big Forbush Decrease (FD) started on June 22nd. and produced by a big CME (Coronal Mass Ejection) held on the Sun several hours before. The FD was observed by most of the neutron monitors and muon detectors existing around the world.

Fig. 7 shows the mean rate, corrected by pressure and temperature, measured by URAGAN, starting several days before the FD; significative decrease is apparent. Also a significative replica was produced around two days later. Fig. 8 shows a muonography giving the arrival angular distribution of muons arriving to the detector during the FD minimum. A sizeable screening in the rate of arrival muons produced by the solar plasma cloud ejected is almost centered in the vertical asymptotic direction below $45^{\circ}$.

Concerning the data taken by TRAGALDABAS, a preliminary analysis has been done using only the information of planes 2 nd. and 4th. and, as a consequence, with a limited angular resolution. Fig. 9 shows the counting rate behaviour in the detector for multiplicity $M=1$ events and for approximately the same period. Fig. 10 shows the counting rates measured at the acceptance angle regions: $\left(0,20^{\circ}\right),\left(20^{\circ}, 30^{\circ}\right)$ and $\left(30^{\circ}, 50^{\circ}\right)$ at four geographic quadrants, where significative differences can be observed.

Waiting for a more detailed analysis, a first glance at the results of both detectors already show interesting effects. The FD has been observed at both locations more or less at the same time. Also both detectors have been sensitive to other small decreases that took place on $\sim$ Jun.16th. and $\sim$ Jul.1st. Another intriguing feature that should be analyzed in the future is the different shape between both FDs. While in Moscow data shows two, or perhaps three, minima of the same size, in the FD observed in Santiago de Compostela there is a main minimum taking place at Jun.25th.. One of the possible reasons of such a difference is the different rigidity thresholds existing in both 


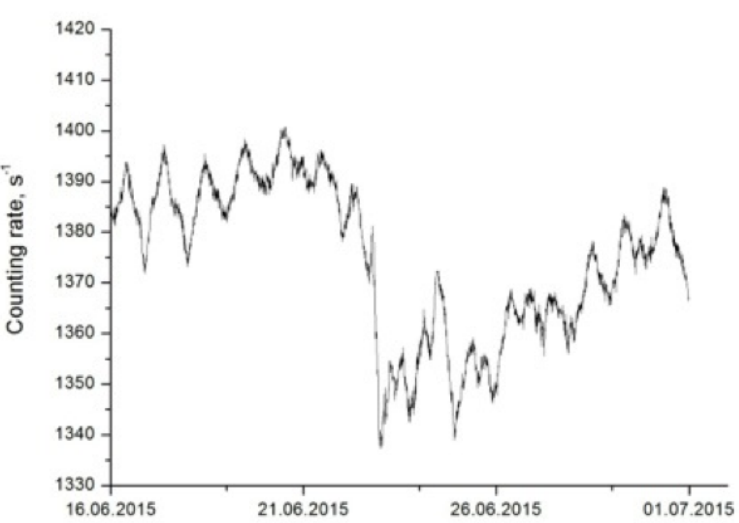

Figure 7: The URAGAN counting rate behavior during the FD in June 22nd. 2015

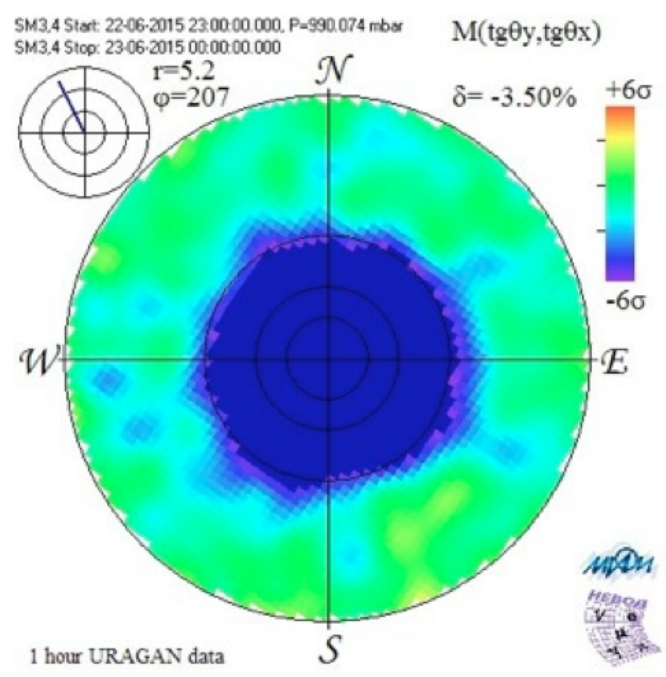

Figure 8: Angular distribution of muons (muonography) measured by URAGAN in the minimum of the FD in June 22nd. showing the big screening produced in the cosmic rays background by a solar plasma cloud.

laboratories: $\sim 2.4 \mathrm{GeV}$ at URAGAN and $\sim 6 \mathrm{GeV}$ at TRAGALDABAS.

\section{Summary}

Both URAGAN and TRAGALDABAS detectors will survey regularly the arrival of cosmic rays with high directional resolution providing a stereographic view of a big portion of the Earth's surface. A first common FD produced as a consequence of the solar activity has been observed recently and is under investigation. A better survey of solar events will allow, in the future, to remove them from the cosmic rays regular flux allowing a better study of other phenomena from atmospheric or interplanetary origin.

\section{References}

[1] NMDB (Neutron Monitor Data Base): http://www.nmdb.eu, GMDN (Global Muon Detector Network): http://crO.izmiran.ru/gmdnet/

[2] V. M. Aynutdinov et al., Neutrino water detector on the Earth's surface (NEVOD), Astrophysics and Space Science 258 (1997) 105.

[3] N.S. Barbashina et al., The URAGAN Wide-Aperture Large-Area Muon Hodoscope Inst. Exp. Tech. 15 (2008) 180-186.

[4] A. Blanco et al., TRAGALDABAS: a new RPC based detector for the regular study of cosmic rays J. of Instrum. 9 (2014) C09027.

[5] J. Belver et al. Analysis of the space-time microstructure of cosmic ray showers using the HADES RPC-TOF wall J. of Instrum. 7 (2012) P10007. 


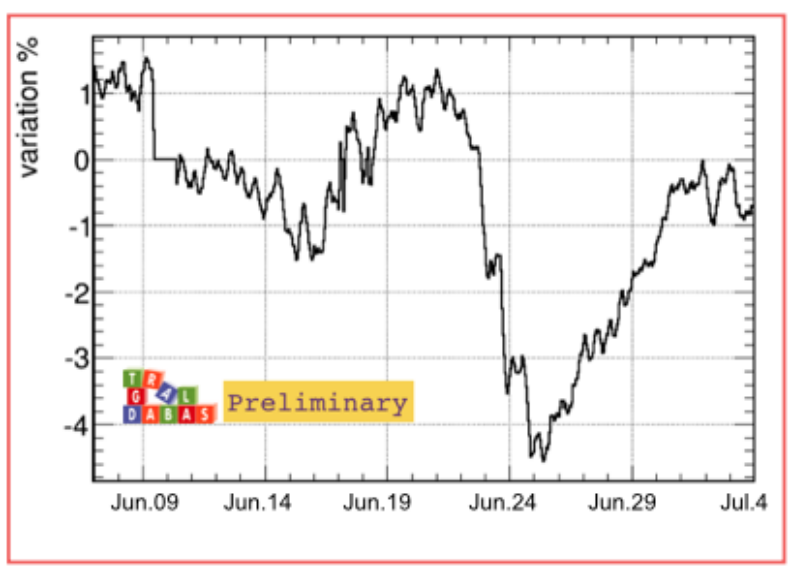

Figure 9: The TRAGALDABAS counting rate behavior during the FD in June 22nd. 2015
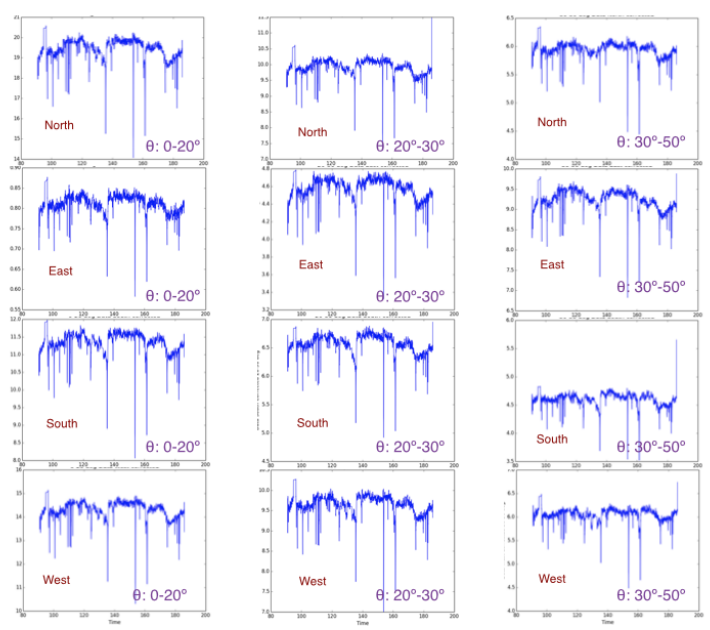

Figure 10: Counting rate measured by TRAGALDABAS at the acceptance regions $\left(0,20^{\circ}\right),\left(20^{\circ}, 30^{\circ}\right)$ and $\left(30^{\circ}, 50^{\circ}\right)$ in the zenith angle and at aproximately the four N, E, S and $\mathrm{W}$ quadrants.

\section{The TRAGALDABAS Collaboration}

Members: H. Alvarez Pol(9), A. Blanco (4), J.J. Blanco (1), J. Collazo (8), J. Díaz Cortés (8), P. Fonte (4), D. García Castro(8), J.A. Garzón (8), A. Gómez-Tato (7), M. González (8), G. Kornakov(6), T. Kurtukian (2), R. Lorenzo (9), J.M. López (8), L. Lópes (4), M. Morales (8), A.Morozova (3), J.C. Mouriño (7), M.A. Pais (3), M. Palka (5), V. Pérez Muñuzuri (10), P. Rey(7), P. Ribeiro (3), M. Seco (9), J. Taboada (10).

Institutions: 1. Univ. Alcalá /Alcalá de Henares, Spain. 2. CEN-Bordeaux, France. 3. CGUCUniv. de Coimbra / Coimbra, Portugal. 4. LIP-Coimbra / Coimbra, Portugal. 5. Jagellionan Univ. /Cracow, Poland. 6. TU-Darmstadt, Germany. 7. CESGA/ S. de Compostela, Spain. 8. LabCAFUniv. S. de Compostela / S. de Compostela, Spain. 9. Particle Phys. Dept. Univ. S. de Compostela / S. de Compostela, Spain. 10. Meteogalicia-Xunta de Galicia / S. de Compostela, Spain. 